\title{
Modeling the properties of plasmaspheric hiss: 1. Dependence on chorus wave emission
}

\author{
Lunjin Chen, ${ }^{1}$ Jacob Bortnik, ${ }^{1}$ Wen Li, ${ }^{1}$ Richard M. Thorne, ${ }^{1}$ and Richard B. Horne ${ }^{2}$ \\ Received 26 September 2011; revised 2 February 2012; accepted 1 March 2012; published 1 May 2012.
}

[1] There is increasing evidence that plasmaspheric hiss is formed by the evolution of a portion of chorus waves that are excited in the plasmatrough and propagate into the plasmasphere. Comparison between the statistical spatial distributions of these two emissions in the morning sector during active times from THEMIS over $\sim 3$ years shows that the two emissions have comparable peak intensities but are distinct in their spatial distributions. We present a modeling study of the hiss spectrum, based on ray tracing, by taking the observed chorus source region as an input in the magnetosphere, which contains cold and suprathermal electrons. Our modeling results show that we are able to reproduce the main features of typical hiss, including the frequency spectrum, wave normal angle and spatial distribution. However, the simulated hiss intensity is weaker ( $\sim 15 \mathrm{~dB}$ less) than the observed intensity, which suggests some modest internal amplification inside the plasmasphere. The responses of hiss to variations in the spatial distribution, wave normal angle distribution and frequency distribution of the source chorus are examined. We find that the majority of hiss formation is due to a small portion of chorus emission originating within $\sim 3 R_{E}$ from the plasmapause, with wave normal directions pointing toward the Earth at an angle of $30^{\circ}-60^{\circ}$, and over a frequency range of $0.1-0.3 f_{\text {ce }}$. If the chorus power is made to increase closer to the plasmapause, the hiss intensity and the peak frequency also increases, which roughly mimics active geomagnetic conditions. Variations of the chorus source distribution do not significantly affect the wave normal angle distribution and frequency distribution of hiss, but does impact the absolute intensity of the resulting hiss.

Citation: Chen, L., J. Bortnik, W. Li, R. M. Thorne, and R. B. Horne (2012), Modeling the properties of plasmaspheric hiss: 1. Dependence on chorus wave emission, J. Geophys. Res., 117, A05201, doi:10.1029/2011JA017201.

\section{Introduction}

[2] Plasmaspheric hiss (PH), as its name suggests, is a structureless, band-limited, natural emission that is generally confined to the plasmasphere [e.g., Russell et al., 1969; Thorne et al., 1973] and associated high density regions such as plumes [Summers et al., 2008]. PH typically occurs in the frequency range from $\sim 200 \mathrm{~Hz}$ to a few $\mathrm{kHz}$, although the main power spectral intensity of hiss is concentrated below $1 \mathrm{kHz}$ [Hayakawa and Sazhin, 1992]. The broad band amplitude of PH depends on the level of geomagnetic activity, ranging from $\sim 10 \mathrm{pT}$ during quite times, up to $>100$ pT during active periods [e.g., Smith et al., 1974; Meredith et al., 2004].

[3] PH plays an important role in controlling the structure and dynamics of the Earth's radiation belts. Pitch angle

\footnotetext{
${ }^{1}$ Department of Atmospheric and Oceanic Sciences, University of California, Los Angeles, California, USA.

${ }^{2}$ British Antarctic Survey, Natural Environment Research Council, Cambridge, UK.

Copyright 2012 by the American Geophysical Union. 0148-0227/12/2011JA017201
}

scattering loss of energetic electrons due to resonance with $\mathrm{PH}$ waves is primarily responsible for the formation of the quiet time, two zone equilibrium radiation belt structure, comprised of the inner $(1.3<L<2.5)$ and outer $(3<L<7)$ belts, and separated by a slot region [e.g., Lyons and Thorne, 1973; Thorne et al., 1973; Albert, 1994; Abel and Thorne, 1998a, 1998b; Meredith et al., 2009]. During storm times, $\mathrm{PH}$ including waves in the plume can efficiently induce precipitation loss of outer zone electrons (100 keV-1 MeV) [Summers et al., 2008; Meredith et al., 2007] and also the outer part of the inner belt [Tsurutani et al., 1975].

[4] A number of ideas have been proposed for explaining the origin of $\mathrm{PH}$. Wave growth calculation inside the plasmasphere yields small gain $(\sim 20 \mathrm{~dB})$ and alone is unlikely to account for the observed hiss emission, which suggests the need for an embryonic source [Huang et al., 1983; Huang and Goertz, 1983; Church and Thorne, 1983]. Lightning has been considered as a potential source [Draganov et al., 1992; Sonwalkar and Inan, 1989; Green et al., 2005], based on the correlation between the geographic distribution of lightning strikes and hiss intensity. However, such correlation occurs only at wave frequencies above $2 \mathrm{kHz}$ while the main frequency band (below $2 \mathrm{kHz}$ ) does not show such 

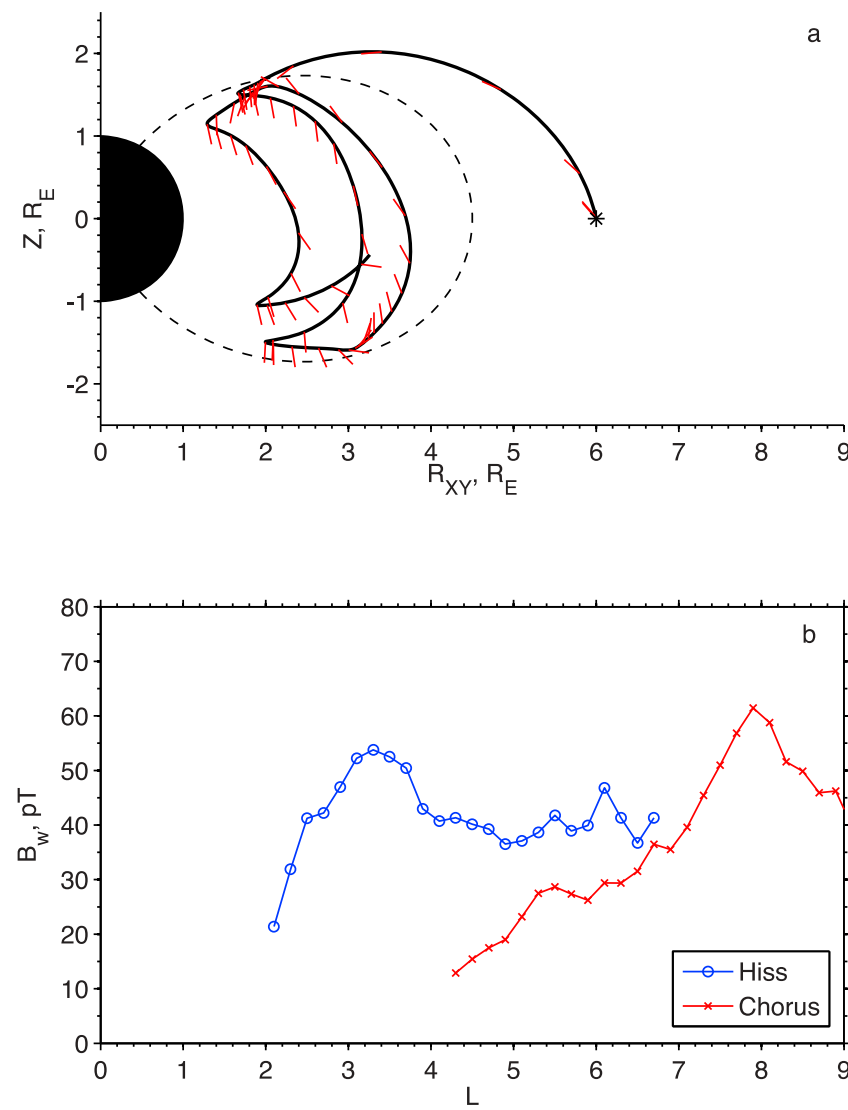

Figure 1. (a) Schematic illustration of the raypath of a chorus wave over 10 seconds, as well as the variation of the wave vector direction during propagation, indicated by the short straight line segments. The dashed line represents the outer edge of the plasmapause $(L=4.5)$. (b) Statistical distribution of chorus and hiss wave amplitude from THEMIS observation $(8 \leq \mathrm{MLT} \leq 12)$.

correlation [Meredith et al., 2006]. Recently, it has been proposed by Bortnik et al. [2008] that PH originates from a portion of chorus emissions, which are excited outside the plasmasphere and are able to propagate into and become trapped inside the plasmasphere (illustrated by Figure 1a). The high-correlation between hiss and chorus wave power, simultaneously observed by two THEMIS spacecraft, lends strong observational support to the idea of chorus as the source of PH [Bortnik et al., 2009]. A similar correlation has also been recently reported from the Cluster spacecraft observations [Wang et al., 2011].

[5] In contrast to $\mathrm{PH}$, chorus is an intense and coherent electromagnetic emission occurring naturally outside the plasmasphere. Chorus is typically observed over the frequency range $0.1-0.8 f_{\text {ce }}$, exhibiting two distinct frequency bands (lower and upper band) separated by $1 / 2 f_{\mathrm{ce}}$. It is generally believed that the generation of chorus waves is due to cyclotron resonance with anisotropic electrons (a few $\mathrm{keV}-100 \mathrm{keV}$ ) [e.g., Jordanova et al., 2010], in the low density plasma trough region [e.g., Meredith et al., 2003; Li et al., 2009]. Like PH, the chorus intensity increases as the level of geomagnetic activity increases [e.g., Li et al., 2009].
The location of peak intensity of chorus waves shows local time dependence, ranging from relatively low $L$-shells $L \sim 5$ on the nightside to $L \sim 8$ on the dayside [Li et al., 2009]. Upper band chorus, and even lower band chorus on the nightside, is confined within a few degrees of the equator, suggesting that they are unlikely to contribute to the origin of $\mathrm{PH}$. However, on the dayside lower band chorus can extend to high latitudes $\left(>35^{\circ}\right)$ [e.g., Meredith et al., 2003; Li et al., 2011b], has strongest intensity, and occurs more frequently than at other local times [Li et al., 2009]. Therefore, the dayside lower-band chorus is considered to be an important source of hiss emissions inside the plasmasphere [Bortnik et al., 2011b]. Lower band chorus usually consists of discrete elements, each of which lasts over timescale $<1 \mathrm{sec}$ and shows fine frequency structure (e.g., falling and rising tones) [e.g., Goldstein and Tsurutani, 1984; Li et al., 2011a], and sometimes short impulsive bursts [e.g., Santolik et al., 2003]. The wave normal angles of chorus near the equatorial source region can vary over a range of values from nearly field-aligned [e.g., Burton and Holzer, 1974; Santolik et al., 2003], to oblique [e.g., Lauben et al., 2002; Tsurutani et al., 2009; Santolik et al., 2009; Breneman et al., 2009]. A recent observational study shows that the wave normal angles of lower band chorus at low latitudes $\left(<15^{\circ}\right)$ are predominately less than $30^{\circ}$ [ Li et al., 2011a, 2011b]. Lower band chorus observations from POLAR also shows another peak occurrence rate for wave normal angles of $50^{\circ} \leq \psi \leq 70^{\circ}$ just off the equator, at a latitude range of $10^{\circ}-25^{\circ}$ [Haque et al., 2010].

[6] Various propagation characteristics of chorus waves have been demonstrated by Bortnik et al. [2011a] and the main observed features of hiss and its wave normal angle distribution are reproduced by our newly developed model based on ray tracing [Bortnik et al., 2011b]. The model considers the magnetosphere as a system box containing a distribution of cold plasma $(<1 \mathrm{eV})$ and suprathermal $(\sim 0.1-10 \mathrm{keV})$ electron fluxes, which determines the chorus wave propagation and damping characteristics respectively. The chorus intensity distribution is provided as an input of the system, and the hiss intensity distribution is obtained as the output. In the present study, a direct comparison between hiss and chorus wave intensity observed in the prenoon sector $8 \leq$ MLT $\leq 12$ from multiple THEMIS spacecraft is made. To model the characteristic hiss spectrum, including the frequency range, the wave normal angle distribution, and the spatial distribution, we use the observed statistical distribution of chorus as the input and compare the output hiss with the statistical distribution of $\mathrm{PH}$ observations. To consider the variability of the chorus emissions mentioned above, we also investigate how the hiss (the output) responds to changes in the chorus distribution (the input) as a function of $L$, wave normal angle, and wave frequency, for a prescribed system, while the responses to the change of the system itself, i.e., the change in the cold plasma density distribution, for a prescribed chorus input is considered in a companion paper by Chen et al. [2012] (hereinafter referred to as paper II). In section 2, the statistical distributions of chorus and hiss wave emission intensity observed by THEMIS, and of suprathermal electron flux are presented. In section 3, a model of the cold plasma density distribution is described. In section 4 , the 
methodology for simulating the hiss spectrum is described. Simulation results are presented in section 5 . The response of hiss wave properties to the variation of chorus distribution is investigated in section 6 , followed by conclusions and a discussion in section 7 .

\section{Chorus and Hiss Emissions and the Suprathermal Electron Flux Distribution From THEMIS}

[7] The THEMIS spacecraft [Angelopoulos, 2008] are well situated to measure chorus and hiss wave intensity in the equatorial magnetosphere. The Search-Coil Magnetometer (SCM) measures the low frequency fluctuation in magnetic field over a frequency range from $0.1 \mathrm{~Hz}$ to $4 \mathrm{kHz}$ [Le Contel et al., 2008; Roux et al., 2008], which covers the frequency range of $\mathrm{PH}$ emission and most of lower band chorus emission. In the present study, filter bank data of wave magnetic field [Cully et al., 2008] from 1 June 2008 to 1 May 2011 from all three inner spacecraft (THEMIS A, D, and E) are analyzed to obtain the root mean square of chorus and hiss wave amplitude in the prenoon sector between 8 and 12 MLT. Chorus wave amplitude is obtained using the same method as in the work by Li et al. [2009], and hiss wave amplitude is calculated from the top three frequency bands $(80-4000 \mathrm{~Hz})$, which cover the essential hiss wave power. Hiss and chorus emissions are distinguished by the total plasma density following the method introduced by Li et al. [2010b], since hiss is predominantly observed in the high density plasmaspherelike region, whereas chorus is typically observed in the low density plasmatrough-like region. Figure $1 \mathrm{~b}$ shows the averaged hiss and chorus wave amplitudes in the prenoon sector $08-12$ MLT in the $L$ bins from 2 to 10 with a bin size 0.2 during active times $\mathrm{AE}^{*}>300 \mathrm{nT}$, where $\mathrm{AE}^{*}$ represents the maximum AE (auroral electrojet) index over the preceding 3 hours. The averaged chorus wave intensity peaks at $L \sim 8$ with a maximum amplitude near $61 \mathrm{pT}$, while the $\mathrm{PH}$ amplitudes inside the plasmapause are about $54 \mathrm{pT}$, with a sharp drop below $L=2.5$. The overlapping region of those two emissions, $4<L<6.5$, is associated with variation of the plasmapause location, potentially due to the variation of geomagnetic activity.

[8] To model the evolution of chorus waves, it is necessary to take into account wave damping along each raypath away from the equatorial generation region, especially Landau damping with suprathermal electrons $(100 \mathrm{eV}-$ $10 \mathrm{keV}$ ) since it is the most severe. The models of suprathermal electron distribution both inside and outside the plasmasphere are constructed based on electron phase space density (PSD) measurement collected by the ElectroStatic Analyzer (ESA) [McFadden et al., 2008] at the six energy channels $108,324,975,2927$, and $8788 \mathrm{eV}$ for $\mathrm{AE}^{*}>300 \mathrm{nT}$, binned in $L$ from 2.5 to 10 at all MLT with bin sizes of $0.5 \mathrm{in} L$ and $1 \mathrm{~h}$ in MLT [see Li et al., 2010a, Figures 3 and 4]. An isotropic power law velocity distribution $A v^{n}$ ( $A$ and $n$ are parameters to be fitted, and $v$ is velocity) is used to fit the averaged PSD data at each of 6 energies in each $L$-MLT bin, similar to the method used by Bortnik et al. [2007], to obtain the global model of suprathermal electron distributions during active times. This distribution is then used to evaluate the wave attenuation along the raypaths.

\section{Cold Electron Density Model}

[9] To study wave propagation in the inhomogeneous magnetosphere, we use a ray tracing code HOTRAY [Horne, 1989] to follow the trajectories of a large group of rays from their source region in the plasma trough. Ray tracing requires analytic models of the magnetic field (we use a dipole field) and plasma density. A simple but realistic and analytic plasma density distribution representative of the dayside meridian plane (MLT $=10)$ is constructed as follows:

$$
N=N_{i}+N_{\mathrm{ps}}(1-g(L))+N_{\mathrm{tr}} g(L)
$$

where the characteristic regions are, the ionosphere $\left(N_{i}\right)$, the plasmasphere $\left(N_{\mathrm{ps}}\right)$ and the trough $\left(N_{\mathrm{tr}}\right)$, which are all included and analytically connected. The transition function $g(L)$ controls the shape of the plasmapause (the transition from the plasmasphere to the plasma trough), defined as,

$$
g(L)= \begin{cases}1 & \text { if } L \geq L_{\mathrm{ppo}} \\ \exp \left(-\left(\frac{L-L_{\mathrm{ppo}}}{L_{\mathrm{ppw}}}\right)^{2}\right) & \text { otherwise }\end{cases}
$$

where $L_{\mathrm{ppo}}$ is the outer edge of plasmapause and $L_{\mathrm{ppw}}$ represents the width of plasmapause.

[10] The low altitude ionosphere $N_{i}$, is characterized by a density peak $\left(n_{\mathrm{F} 2}\right)$ at the F2 location $r_{\mathrm{F} 2}$, and is modeled as a function of geocentric distance $r$ :

$$
N_{i}(r)=\left\{\begin{array}{ll}
n_{\mathrm{F} 2} \exp \left(-\left(r-r_{\mathrm{F} 2}\right)^{2} / \Delta_{\mathrm{r} 1}^{2}\right) & \text { if } r \leq r_{\mathrm{F} 2} \\
n_{\mathrm{F} 2} \exp \left(-\left(r-r_{\mathrm{F} 2}\right)^{2} / \Delta_{\mathrm{r} 2}^{2}\right) & \text { if } r>r_{\mathrm{F} 2}
\end{array} .\right.
$$

Different spatial scales over which density decreases away from the F2 peak are applied, i.e., $\Delta_{\mathrm{r} 1}=140 \mathrm{~km}, \Delta_{\mathrm{r} 2}=$ $500 \mathrm{~km}$, typical F2 peak is assumed to have $n_{\mathrm{F} 2}=10^{6} \mathrm{~cm}^{-3}$ at $r_{\mathrm{F} 2}=350 \mathrm{~km}+1 R_{E}$ [Richards and Torr, 1985; Reinisch et al., 2007], where the Earth's radius $R_{E}=6380 \mathrm{~km}$.

[11] The plasmaspheric density $N_{\mathrm{ps}}$ is constructed as:

$$
N_{\mathrm{ps}}(L, r)=n_{\mathrm{eps}}(L)\left(\frac{L}{r}\right)^{\alpha_{\mathrm{ps}}(L)}
$$

where the equatorial density inside the plasmasphere $n_{\mathrm{eps}}(L)=10^{-0.3145 L+3.9043} \mathrm{~cm}^{-3}$ [Carpenter and Anderson, $1992]$, and variation along a field line is assumed to have a form of $(L / r)^{\alpha_{\mathrm{ps}}}$ [Denton et al., 2002, 2006]. $\alpha_{\mathrm{ps}}(L)$ is chosen such that $N_{\mathrm{ps}}(L, r=1)=n_{0 \mathrm{ps}}$, independent of $L$, i.e., $N_{\mathrm{ps}}$ tends to merge toward a constant $n_{0 \mathrm{ps}}$ at $r=1$ along any field line inside the plasmasphere. $n_{0 \mathrm{ps}}=5000 \mathrm{~cm}^{-3}$ is used, which yields $\alpha_{\mathrm{ps}} \sim 1.5$ inside the plasmasphere, comparable to that found by Denton et al. [2006].

[12] A similar distribution is applied to obtain the plasma trough density $N_{\text {tr: }}$ :

$$
N_{\text {tr }}(L, r)=n_{\text {etr }}(L)\left(\frac{L}{r}\right)^{\alpha_{\mathrm{tr}}(L)},
$$



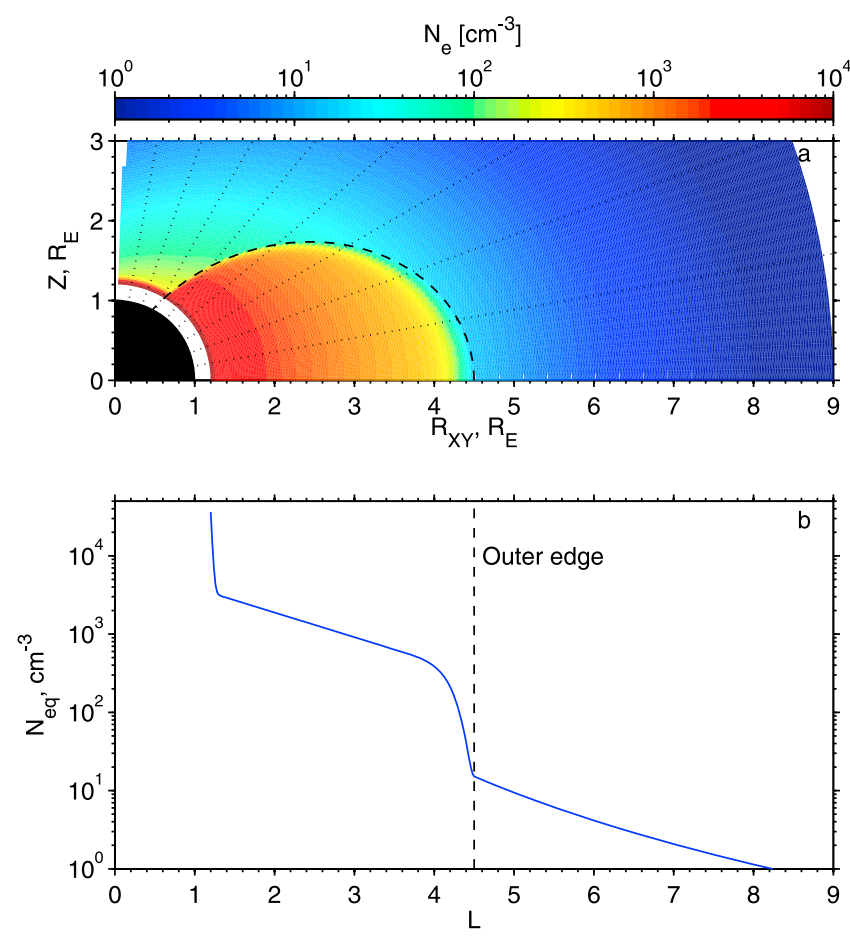

Figure 2. (a) Electron density distribution in the meridian plane (MLT $=10)$ and (b) the corresponding equatorial density profile. The outer edge of the plasmapause is denoted by the vertical dashed line.

where the equatorial density in the plasma trough $n_{\mathrm{etr}}(L)=13200 L^{\beta} \mathrm{cm}^{-3}$ and trough slope index $\beta=-4.5$ [Carpenter and Anderson, 1992], and $\alpha_{\mathrm{tr}}(L)$ is chosen such that $N_{\text {tr }}\left(L, r=1 R_{E}\right)=n_{0 \text { trr }}$, independent of $L$, i.e., $N_{\text {tr }}$ tends to merge toward $n_{0 \text { tr }}$ at $r=1$ along any field line in the plasma trough. $N_{0 \mathrm{tr}}=500 \mathrm{~cm}^{-3}$ is used, giving $\alpha_{\mathrm{tr}} \sim 2.5$, a value typical of the plasma trough [Denton et al., 2002].

[13] Our nominal density distribution uses the parameter values listed above together with $L_{\mathrm{ppw}}=0.7$, and $L_{\mathrm{ppo}}=4.5$. The corresponding meridian distribution and equatorial profile of plasma density are shown in Figures $2 a$ and $2 b$, respectively. This nominal density distribution will be used throughout this study. The density model used in our study provides a simple, analytical density distribution (which is required for ray tracing), where the equatorial density distribution is realistic, based on the statistical results of Carpenter and Anderson [1992], and the distribution along the field line is reasonably well represented according to previous studies [e.g., Denton et al., 2002]. Our density model consists of four characteristic regions in the inner magnetosphere, the ionosphere, plasmasphere, plasmapause, and trough, whose shapes can be adjusted easily to represent background plasma density distribution at different conditions. Previous studies demonstrated the variability of the trough density [e.g., Harris, 1974; Kitamura et al., 2009], and the variability of the plasmapause location with geomagnetic activity [e.g., Grebowsky, 1970; Chappell et al., 1970; Goldstein et al., 2003]. Our density model is able to vary trough density and the plasmapause independently, which is very important since it gives us an opportunity to study separately the effect of trough density on the chorus wave propagation into the plasmasphere and the effect of the plasmapause on the distribution of chorus waves that are able to gain access into the plasmasphere. These effects are presented in paper II. The effect of a fine density structure associated with the plasmapause [e.g., Fu et al., 2010] is beyond the scope of our current study. The density model used in this study is rather simpler than the density model used previously, e.g., the diffusive equilibrium model [Bortnik et al., 2011a], but is able to reproduce a similar group of chorus rays that penetrate into the plasmasphere from outside and thus contribute to the hiss emission.

\section{Hiss Modeling}

[14] The methodology of modeling the hiss spectrum is based on a ray tracing technique, explained in detail in our previous work [Bortnik et al., 2011b]. Here we review the modeling briefly. We launch rays from the equator outside the plasmapause in the meridian plane corresponding to MLT $=10$, to represent the excited chorus rays, with a range of $L$-shell, $L_{\mathrm{ppo}} \leq L_{i} \leq 9$ with spacing $\Delta L=0.1$, the entire lower band wave frequency $0.05 f_{\text {ce }} \leq f_{i} \leq 0.5 f_{\text {ce }}$ with spacing $\Delta f=0.05 f_{\mathrm{ce}}$, and all wave normal angles $\psi_{i}$ within the resonance cone angle $\left(\left|\cos \psi_{i}\right|>\cos \psi_{\text {res }} \equiv f_{i} / f_{\text {ce }}\right)$ with spacing $\Delta \psi=1^{\circ}$. Note that positive and negative signs of $\psi$ denote ani-Earthward and Earthward pointing wave normals, respectively. In total $\sim 10^{5}$ rays are traced. Each chorus ray is assigned with an initial power according to

$$
\begin{aligned}
\Delta P_{w}\left(L_{i}, f_{i}, \psi_{i}\right)= & B_{w}^{2}\left(L_{i}\right) A_{L} \exp \left(-\frac{\left(X_{i}-X_{m}\right)^{2}}{X_{w}^{2}}\right) \\
& \cdot \exp \left(-\frac{\left(f_{i}-f_{m}\right)^{2}}{f_{w}^{2}}\right) \Delta L \Delta f \Delta \psi,
\end{aligned}
$$

where $X_{i}=\tan \psi_{i}, X_{m}=\tan \psi_{m}, X_{w}=\tan \psi_{w}, \psi_{m}$ and $\psi_{w}$ are wave normal peak and width respectively, $f_{m}$ and $f_{w}$ are the frequency peak and width respectively, $B_{w}^{2}(L)$ is wave magnetic field power as a function of $L$, and $A_{L}$ is the normalization factor such that

$$
A_{L} \int_{f_{1}}^{f_{2}} d f \int d \psi \exp \left(-\frac{\left(X_{i}-X_{m}\right)^{2}}{X_{w}^{2}}\right) \exp \left(-\frac{\left(f_{i}-f_{m}\right)^{2}}{f_{w}^{2}}\right)=1
$$

with $f_{1}$ and $f_{2}$ denoting the lower and upper cutoff frequency of lower band chorus respectively.

[15] In addition to computing the wave propagation, we also carefully treat the wave damping primarily due to Landau resonance with suprathermal electrons $(0.1-10 \mathrm{keV})$ after the waves are generated. We use a modeled suprathermal electron distribution both inside and outside the plasmasphere at MLT $=10$ as described in section 2 . Subsequently, these rays are subject to Landau damping, the rate of which is evaluated according to Chen et al. [2010, equation (2)]. Rays are terminated when the power attenuation exceeds $40 \mathrm{~dB}$ or the ray refractive index $n$ exceeds $10^{3}$, where wave phase speed becomes comparable to the thermal speed of plasmaspheric electrons (a few eV) and thus cold plasma ray tracing is not valid [Bortnik et al., 2011b]. A geometric contraction factor of ray power variation to represent the convergence of magnetic field lines is also 

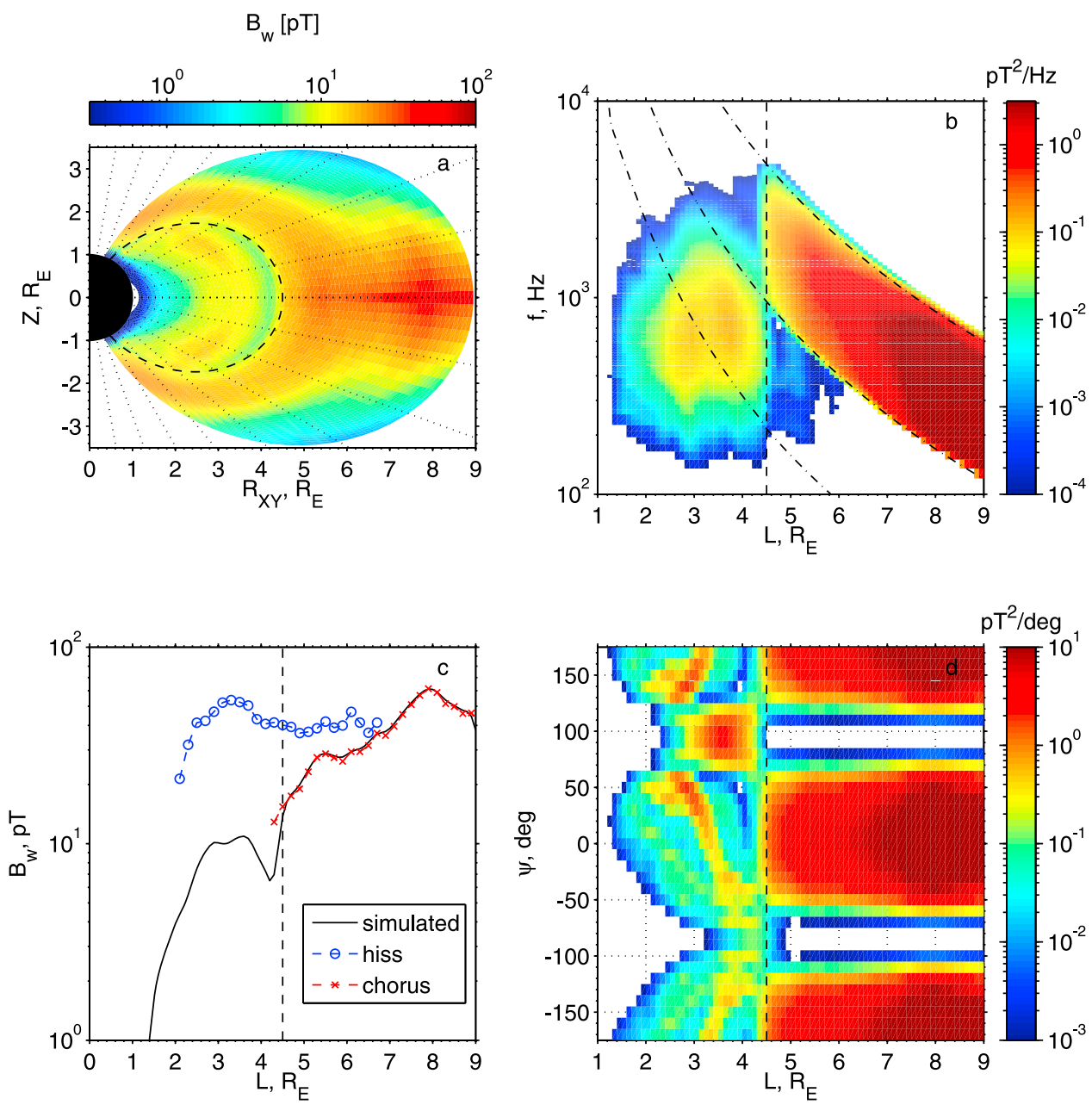

Figure 3. (a) Spatial distribution of the modeled wave amplitudes in the meridian plane both inside and outside the plasmapause. (b) The equatorial wave frequency spectrum. (c) The equatorial wave amplitude profile. The red crosses are statistical chorus wave amplitudes (Figure 1b), which act as the source of the wave emission. The blue circles are statistical hiss wave amplitude (Figure 1b). (d) The equatorial wave normal angle spectrum. Dashed line in Figure 3a denotes the field line of the outer edge of plasmapause $(L=4.5)$. Dotted lines in Figure 3a represent contours of constant latitude from $-80^{\circ}$ to $+80^{\circ}$ with a spacing of $10^{\circ}$. Vertical dashed lines in Figures $3 \mathrm{~b}-3 \mathrm{~d}$ represent the outer edge of the plasmapause. The dotdashed lines in Figure $3 \mathrm{~b}$, from top to bottom, denote $0.5 f_{c e}, 0.1 f_{c e}$, and $f_{\text {lhr }}$ respectively.

taken into account [Bortnik et al., 2011b]. It should be noted that the chorus generation is not considered in the present study, instead the generated chorus wave power is specified according to equation (6). Based on tracing the raypath, the wave normal, and the wave power of all the rays, we reconstruct the wave power as $P_{w}(L, \lambda, f, \psi)$ in units of $\mathrm{pT}^{2} / \mathrm{Hz} / \mathrm{deg}$, as a function of spatial coordinates $L$ and latitude $\lambda$, wave normal angle $\psi$ and wave frequency $f$.

[16] We choose as a nominal spectral distribution $f_{1}=0.1 f_{\text {ce }}, f_{2}=0.5 f_{\text {ce }}, \psi_{m}=0^{\circ}, \psi_{w}=45^{\circ}, f_{m}=0.25 f_{\text {ce }}$ and $f_{w}=0.15 f_{\text {ce }}$, based on chorus observations [see Bortnik et al. $2011 \mathrm{~b}$, and reference within]. Figure 3 shows the results using as an input the chorus with the above nominal spectral distribution and the statistical spatial distribution from THEMIS (red in Figure 1b). Figure 3a shows the simulated spatial distribution of wave amplitudes in $\mathrm{pT}, \sqrt{\iint P_{w} d f d \psi}$. Clearly as chorus waves propagate away from the equatorial generation region, the wave power decreases due to Landau damping. Although the chorus intensity peaks at $L \sim 8$ at the equator, at high latitudes $\left(>\sim 20^{\circ}\right)$ chorus intensity is stronger at the inner region of the plasma trough $(L<\sim 7)$ because of stronger Landau damping due to an increase in suprathermal electron flux at the higher $L$-shells. A secondary wave power peak is apparent at high latitudes and is associated with the spatial convergence of the magnetic field. Chorus wave power is able to access to the plasmapause mostly over a range of latitudes $30^{\circ}<\lambda<60^{\circ}$ and $-60^{\circ}<\lambda<-30^{\circ}$, and thus contribute to the subsequent hiss formation. This is consistent with the observation on OGO 3 [Russell et al., 1969], identifying the region above $L=6$ near magnetic latitudes of $45^{\circ}$ as a source of steady noise. The entrance at high latitude results in a minimum of wave power just inside the outer edge of the plasmapause near the equatorial region $|\lambda|<\sim 30^{\circ}$. The simulated equatorial wave amplitude profile (black line in Figure $3 \mathrm{c}$ ), $\sqrt{\iint P_{w} \mathrm{I}_{\lambda=0} d f d \psi}$, also shows a peak hiss amplitude of $10 \mathrm{pT}$ at $L \sim 3.6$ with a significant 

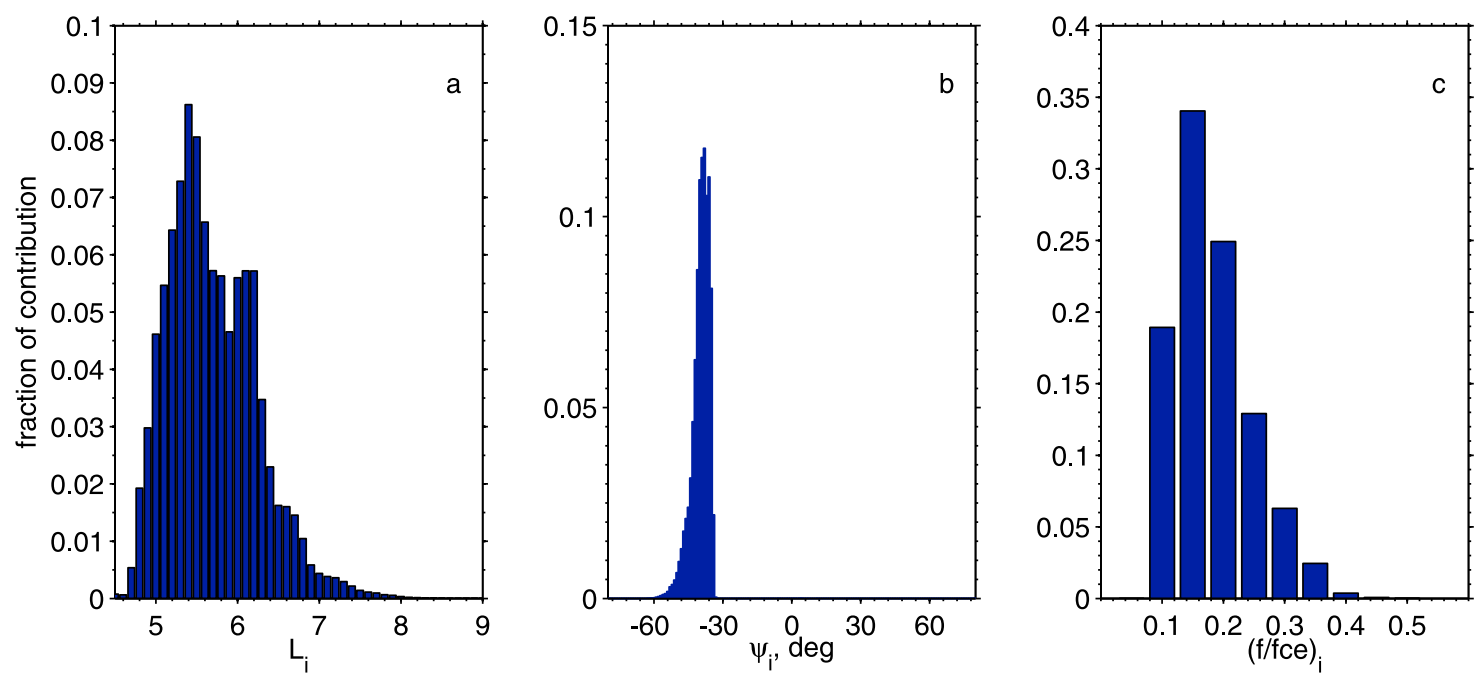

Figure 4. The fractional contribution of chorus rays to the hiss emission within the plasmasphere ( $L \leq 4.3$ ) due to (a) varying launch locations $L_{i}$, (b) varying the initial wave normal angle $\psi_{i}$, and (c) varying the initial normalized wave frequency $\left(f / f_{\mathrm{ce}}\right)_{i}$.

decrease at $L \sim 2$, consistent with the observed hiss profile. However, the modeled wave amplitude is a factor of $\sim 5$ lower than the observed peak hiss wave amplitude $54 \mathrm{pT}$ ( $\sim 15 \mathrm{~dB}$ less in power), which we attribute primarily to the lack of consideration of wave growth inside the plasmasphere. The path-integrated gain of hiss inside the plasmasphere can be up to $\sim 20 \mathrm{~dB}$, based on the calculation performed by Church and Thorne [1983], which is too small to excite the hiss from the thermal noise inside the plasmasphere but could sufficiently account for the discrepancy between observed and simulated hiss wave amplitude. The frequency spectrum from our simulation agrees well with the typical hiss frequency range $\sim 200 \mathrm{~Hz}$ to $2 \mathrm{kHz}$ (Figure $3 \mathrm{~b}$ ). In addition, our model can also simulate the wave normal angle distribution of hiss (Figure 3d), showing near fieldaligned distribution at lower $L$ and oblique distribution in the outer plasmasphere. Characteristics of wave frequency and the wave normal angle spectrum off the equator have been shown by Bortnik et al. [2011b]. We concentrate on the equatorial emission only in this study. As pointed out by Bortnik et al. [2011b], there is a symmetry about $\psi= \pm 90^{\circ}$ in the equatorial wave normal angle distribution due to the symmetry of northward and southward propagation of chorus rays and the symmetry of dipole field.

[17] To examine how chorus emissions contribute to the equatorial hiss emission, we first define the total equatorial hiss emission $I$ as the integration of the wave power over the spatial region inside the plasmasphere $(1 \leq L \leq 4.3$ where the plasma density is greater than $\sim 100 \mathrm{~cm}^{-3}$ ):

$$
I=\int_{L=1}^{4.3} \int_{f} \int_{\psi} P_{\mathrm{eq}}(L, f, \psi) d L d f d \psi,
$$

where $P_{\text {eq }}=P_{w}(L, \lambda=0, f, \psi)$ is the equatorial power density in units of $\mathrm{pT}^{2} / \mathrm{Hz} / \mathrm{deg}$. The relative fraction of $I$ contributed by various groups of chorus rays is then calculated. Figures $4 \mathrm{a}-4 \mathrm{c}$ show the relative fraction of the contribution by chorus rays of varying initial launch location $L_{i}$, those of varying initial wave normal angle $\psi_{i}$ and those of varying initial normalized wave frequency $f_{i} / f_{\text {ce }}$, respectively. Clearly the dominant source of hiss is from chorus within $\sim 3 R_{E}$ of the outer edge of plasmapause $(\sim 95 \%)$, with wave normal angles $-60^{\circ}--30^{\circ}(\sim 100 \%)$, and normalized wave frequency $f / f_{\text {ce }} 0.1-0.3(\sim 98 \%)$. Note that rays launched at $L>8$, although carrying the largest wave power, can not access to the plasmasphere (Figure $3 \mathrm{a}$ ) because of the severe Landau damping at higher $L$ and the longer propagation path that the rays must execute before entering into the plasmapause. On the other hand, the rays launched too close to the plasmapause $(L<5)$ are mostly trapped near the plasmapause and thus can not penetrate into the inner region of the plasmasphere. While the precise fractional contributions depend on the adopted model of the chorus spatial and spectrum distribution, the results shown in Figure 4 are representative of the general trend. The sensitivity of the access of chorus waves to the plasmasphere is explored below.

\section{Hiss Response to the Variations in the Chorus Source Distribution}

[18] A sensitivity study has been performed to investigate how the properties of hiss respond to changes in the chorus source characteristics. Instead of the complex spatial distribution exhibited in the statistical observations (Figure 1b), a Gaussian distribution in $L$ is adopted in our modeling for simplicity,

$$
B_{w}(L)=B_{\text {peak }} \exp \left(-\frac{\left(L-L_{\text {peak }}\right)^{2}}{L_{w}^{2}}\right)
$$

with $L_{w}=0.15, B_{\text {peak }}=100 \mathrm{pT}$ [Bortnik et al., 2011b], and a peak location $L_{\text {peak }}$ which is allowed to vary. We adopt a peak chorus amplitude of $100 \mathrm{pT}$, which is more representative of storm time periods than $61 \mathrm{pT}$ average observed by THEMIS in recent years (Figure 1b), which was generally obtained under relatively quiet geomagnetically conditions. Because of linear summation of wave power, $P_{w}$ scales as $B_{\text {peak }}^{2}$, and hence hiss amplitude scales as $B_{\text {peak }}$. The chorus 

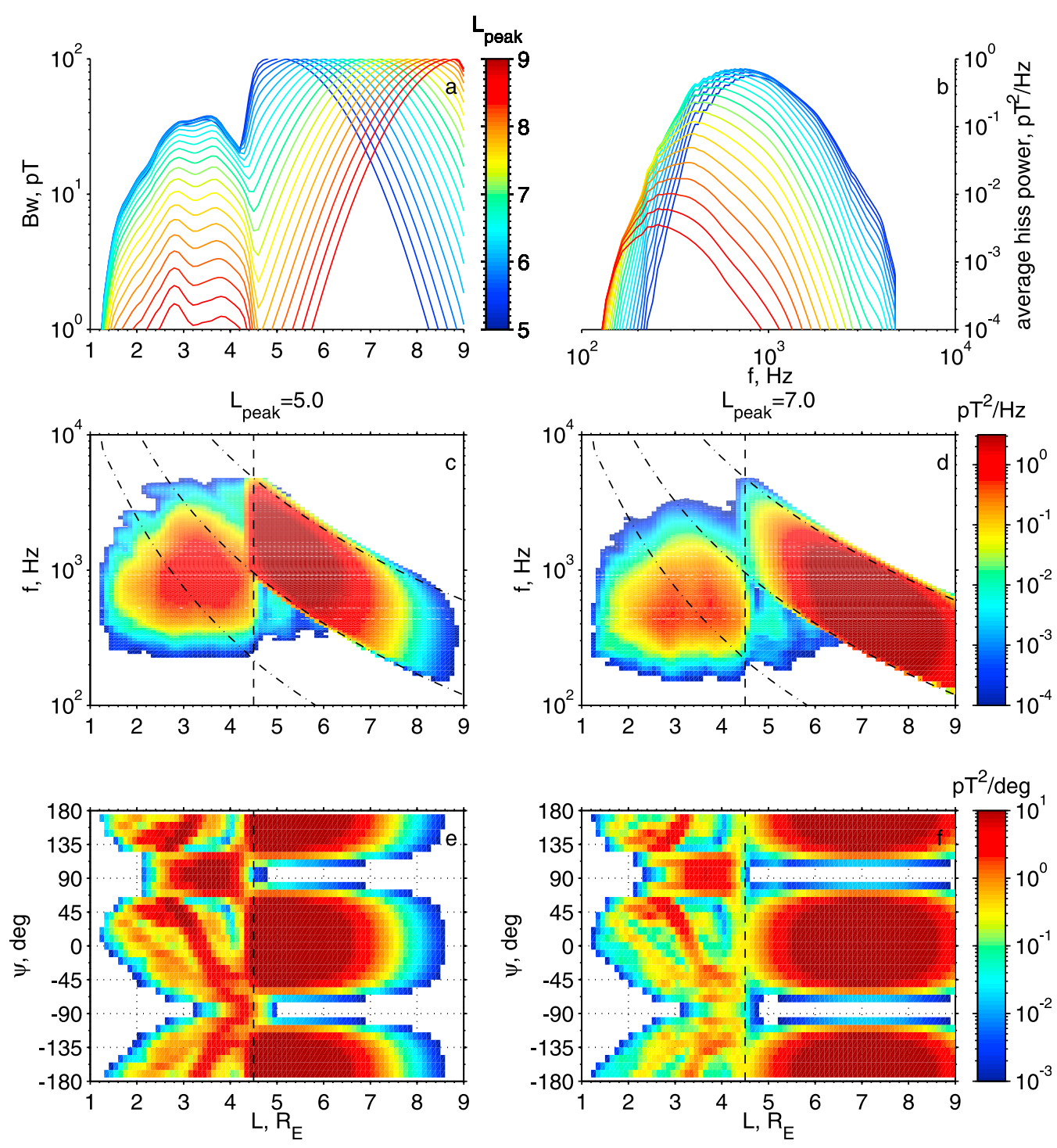

Figure 5. The dependence of equatorial hiss emission on the spatial distribution of chorus emission. (a) The equatorial hiss amplitude profile as a function of $L$ and (b) averaged hiss power spectral intensity as a function of frequency, for varying peak locations of chorus amplitude profile (color-coded). (c and e) The frequency and wave normal distribution of equatorial wave emission for $L_{\text {peak }}=5$. ( $\mathrm{d}$ and f) The same format for $L_{\text {peak }}=7$ (the nominal case). The three black dot-dashed lines in Figures $5 \mathrm{c}$ and $5 \mathrm{~d}$ represents $0.5 f_{\mathrm{ce}}, 0.1 f_{\mathrm{ce}}$, and $f_{\mathrm{LHR}}$. Vertical black lines indicate the outer edge of the plasmapause.

distribution is parameterized by the seven quantities in this study, $L_{\text {peak }}$ in radial distribution, $\psi_{m}$ and $\psi_{w}$ in wave normal angle distribution, and $f_{m}, f_{w}, f_{1}$ and $f_{2}$ in the wave frequency distribution. We choose the nominal chorus distribution to be $L_{\text {peak }}=7, \psi_{m}=0^{\circ}, \psi_{w}=45^{\circ}, f_{m}=0.25 f_{\text {ce }}, f_{w}=0.15 f_{\text {ce }}$, $f_{1}=0.1 f_{\mathrm{ce}}$, and $f_{2}=0.5 f_{\mathrm{ce}}$, and then vary one of these seven parameters to investigate how equatorial hiss responds to the changes.

\subsection{Variation in $L_{\text {peak }}$}

[19] Figure 5 shows the response of the hiss distribution to the variation of $L_{\text {peak }}$ from 5 to 9 . Variations of the spatial distribution of simulated equatorial wave amplitude $\sqrt{\iint P_{\mathrm{eq}} d f d \psi}$ are shown in Figure 5a, and the frequency spectrum averaged inside the plasmasphere over $1<L<4.3$, $\left\langle\int P_{\mathrm{eq}} d \psi\right\rangle_{\text {ave }}$ are shown in Figure 5b. Also shown are simulation results with $L_{\text {peak }}=5$ of the equatorial frequency spectrum $\int P_{\text {eq }} d \psi$ (Figure 5c) and wave normal distribution $\int P_{\text {eq }} d f$ (Figure 5e), and similar results with $L_{\text {peak }}=7$ (Figures $5 \mathrm{~d}$ and $5 \mathrm{f}$ ). It is clear from Figure $5 \mathrm{a}$ that as the chorus source moves closer to the plasmapause (from $L_{\text {peak }}=9$ to $L_{\text {peak }}=5$ ), the amplitude of hiss can increase by an order of magnitude from a peak value of $\sim 2 \mathrm{pT}$ to $\sim 30 \mathrm{pT}$, while the hiss frequency spectrum shifts to higher frequencies (Figure 5b), due to the increase in chorus power at small $L$ (and consequently larger $f_{\text {ce }}$ ). The peak frequency of averaged hiss increases from $\sim 250 \mathrm{~Hz}$ for $L_{\text {peak }}=9$ to $\sim 900 \mathrm{~Hz}$ for $L_{\text {peak }}=5$. In contrast, the wave normal distribution does not show any significant variation, although the absolute intensity varies (Figures 5e and 5f). 

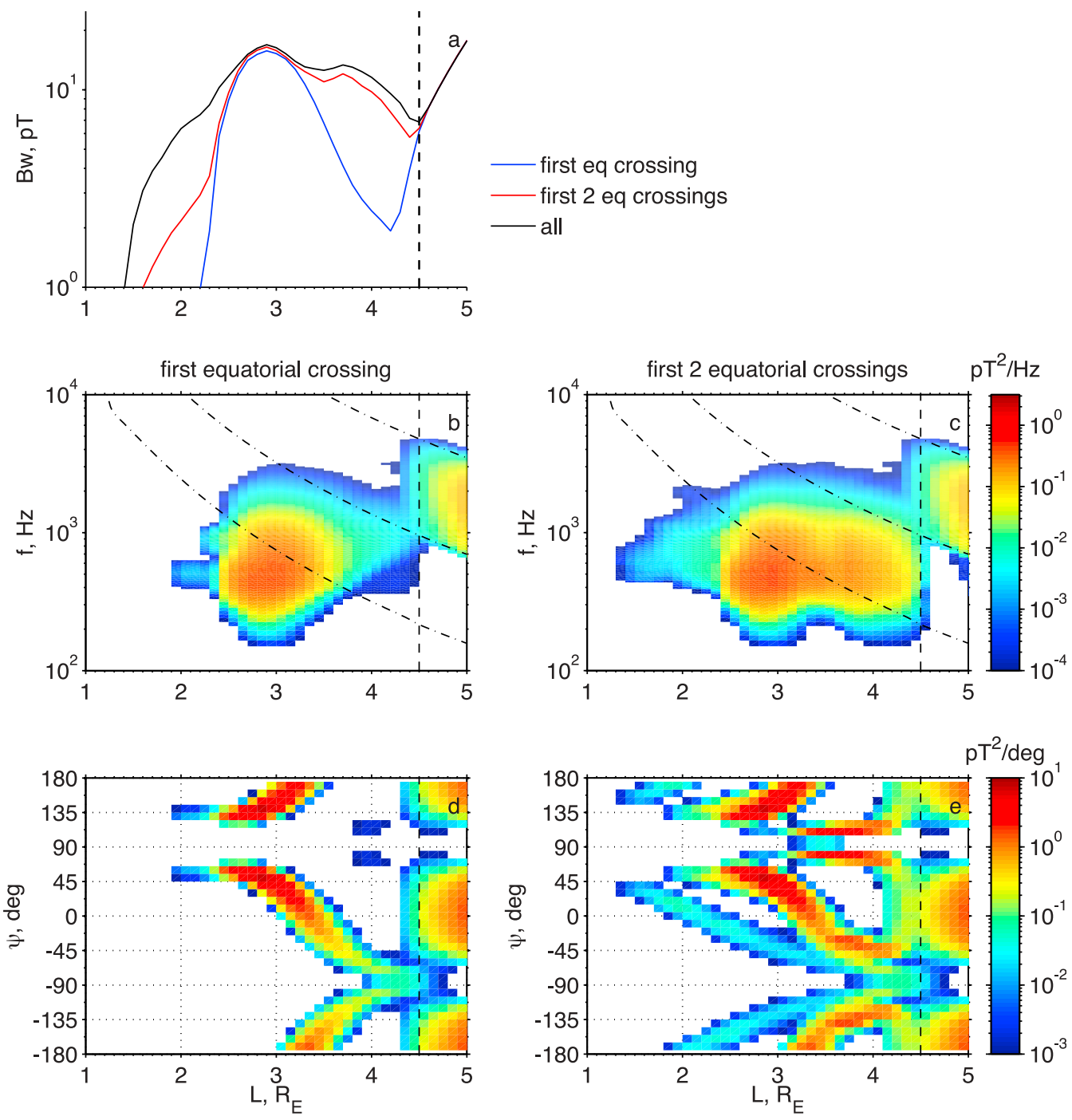

Figure 6. (a) Analysis of the hiss two peak formation: equatorial hiss emission for the first equatorial crossing (blue line), for the first two equatorial crossings (red line) and for all the equatorial crossings (black line). The (b) frequency and (d) wave normal angle spectrum for the first equatorial crossing. (c and e) The same spectrum as in Figures $6 \mathrm{~b}$ and $6 \mathrm{~d}$ except for the first two equatorial crossings, and Figures $5 \mathrm{~d}$ and $5 \mathrm{f}$ for all the equatorial crossings. Black vertical dashed lines denote the outer edge of the plasmapause. Black dot-dashed lines represent $0.5 f_{\text {ce }}, 0.1 f_{\text {ce }}$ and $f_{\text {LHR }}$.

[20] Also evident in these simulations are two consistent peaks in the hiss intensity inside the plasmasphere $(L<4.3)$, at $L \sim 2.8$ and $L \sim 3.8$, in Figure 5a, which are present regardless of the value of $L_{\text {peak. }}$. Such a two peak distribution is also observed in CRRES spacecraft data [e.g., Bortnik et al., 2011b, Figure 5]. To investigate how these two peaks form, we analyze the equatorial emission for the nominal case $\left(L_{\text {peak }}=7\right)$. Figure 6a shows the equatorial wave amplitude of hiss for 3 cases: (i) when only the first equatorial crossing of chorus rays is included (blue line), (ii) when the first two equatorial crossings are included (red line) and (iii) when all equatorial crossings are included (black line) until waves damp out. The frequency spectrum and the wave normal distribution corresponding to the first equatorial crossing are shown in Figures $6 \mathrm{~b}$ and $6 \mathrm{~d}$, those corresponding to the first two equatorial crossings shown in
Figures $6 \mathrm{c}$ and $6 \mathrm{e}$, and those corresponding to all the equatorial crossings shown in Figures $5 \mathrm{~d}$ and $5 \mathrm{f}$. The hiss wave power for the first equatorial crossing is limited to an $L$-shell range of $2<L<4$, with an intensity peak at $L \sim 2.8$ (corresponding to the inner peak). Waves are confined to intermediate wave normal angles $\sim 45^{\circ}$ and $\sim 135^{\circ}$ (Figure 6d), and occur over a broad frequency range $200 \mathrm{~Hz}<f<3 \mathrm{kHz}$ (Figure 6b). A comparison of Figures $6 \mathrm{~d}$ and $6 \mathrm{e}$ indicates that the second equatorial crossing adds field-aligned waves at inner $L$-shells $(L<3)$ and oblique waves at outer $L$-shell $(L>3.5)$. Over multiple equatorial crossings, the wave intensity spreads both outward and inward from the inner peak due to the evolution of emissions trapped inside the plasmasphere, and tends to accumulate at $L \sim 3.8$ due to propagation characteristics associated with magnetospheric reflection, i.e., whistler mode waves tend to settle down in a $L$-shell where $f$ is just 

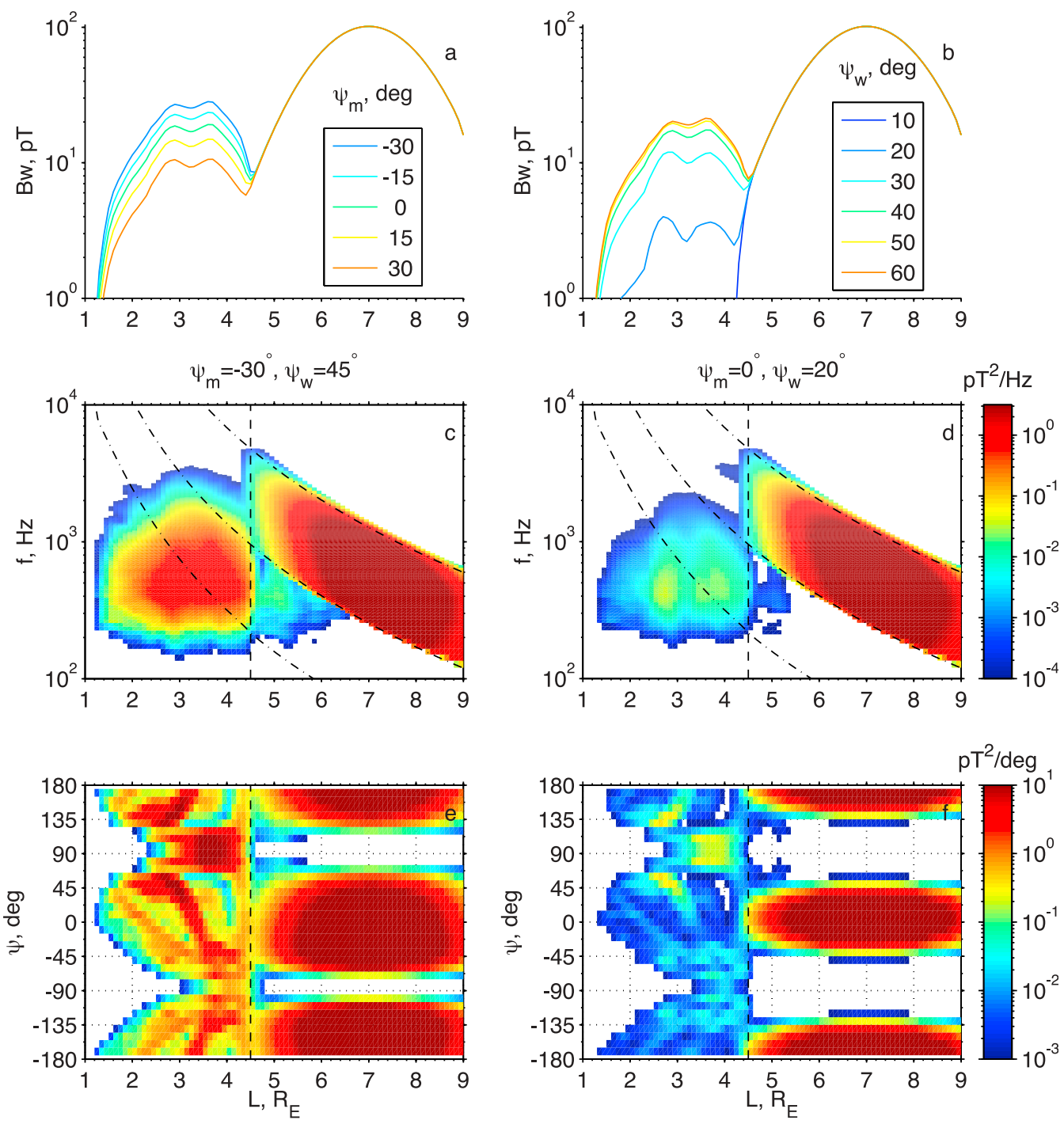

Figure 7. The dependence of equatorial hiss emission on the wave normal angle distribution of chorus emission. (a) The equatorial hiss amplitude profile for varying chorus peak wave normal angle $\psi_{m}$ (color-coded). The (c) frequency and (e) wave normal angle spectrum of equatorial wave emission for $\psi_{m}=-30^{\circ}$. (b, d, and f) The same format as Figures 7a, 7c, and 7e except for varying chorus wave normal angle width $\psi_{w}$. Figures $7 \mathrm{~d}$ and $7 \mathrm{f}$ show the frequency and wave normal angle spectrum of equatorial wave emission for $\psi_{w}=20^{\circ}$. The spectrum for the nominal case is shown in Figures $5 \mathrm{~d}$ and $5 \mathrm{f}$ as comparison.

above the equatorial $f_{\mathrm{LHR}}$ and $\psi$ approaches $90^{\circ}$. The settling of waves contributes to the formation of the outer peak. The inner peak of the hiss distribution is a measure of the initial accessibility of chorus rays into the equatorial plasmasphere from the first high latitude reflection, while the remainder of the emission is simply due to the evolution of the waves due to confinement inside the plasmasphere.

\subsection{Variation in Chorus Wave Normal Distribution}

[21] The response of hiss to variations of the chorus wave normal angle distribution is investigated in Figure 7. Variations of the equatorial wave amplitude due to the change of the wave normal angle peak $\psi_{m}$ and wave normal angle width $\psi_{w}$ are shown in Figures $7 \mathrm{a}$ and $7 \mathrm{~b}$ respectively. The frequency spectrum and wave normal distribution for the case $\psi_{m}=-30^{\circ}$ and $\psi_{w}=45^{\circ}$ are shown in Figures $7 \mathrm{c}$ and $7 \mathrm{e}$, while those for the case $\psi_{m}=0^{\circ}$ and $\psi_{w}=20^{\circ}$ are shown in
Figures $7 \mathrm{~d}$ and $7 \mathrm{f}$. As the chorus peak wave normal direction shifts toward the Earth (more negative $\psi_{m}$ ), the hiss wave power is enhanced (Figure 7a). When chorus wave normal distribution becomes narrower and thus more confined to the field aligned direction, the resulting hiss is subject to significant suppression (Figure $7 \mathrm{~b}$ ). These variations are consistent with Figure $4 \mathrm{~b}$, showing that the dominant contribution of hiss intensity comes from those chorus rays in the source region with wave vectors inclined over the range from $-30^{\circ}$ to $-60^{\circ}$ to the ambient magnetic field, and it is the power weighting in this critical range of wave normals that determines the amount of power that is able to enter the plasmasphere and evolves into hiss. Compared with the results of the nominal case (Figures $5 \mathrm{~d}$ and $5 \mathrm{f}$ ), the frequency spectrum and wave normal distribution for both $\psi_{m}=-30^{\circ}$ (Figures $7 \mathrm{c}$ and 7e) and $\psi_{w}=20^{\circ}$ (Figures 7d and 7f) look fairly similar, except for the difference in the absolute intensity. In all these 

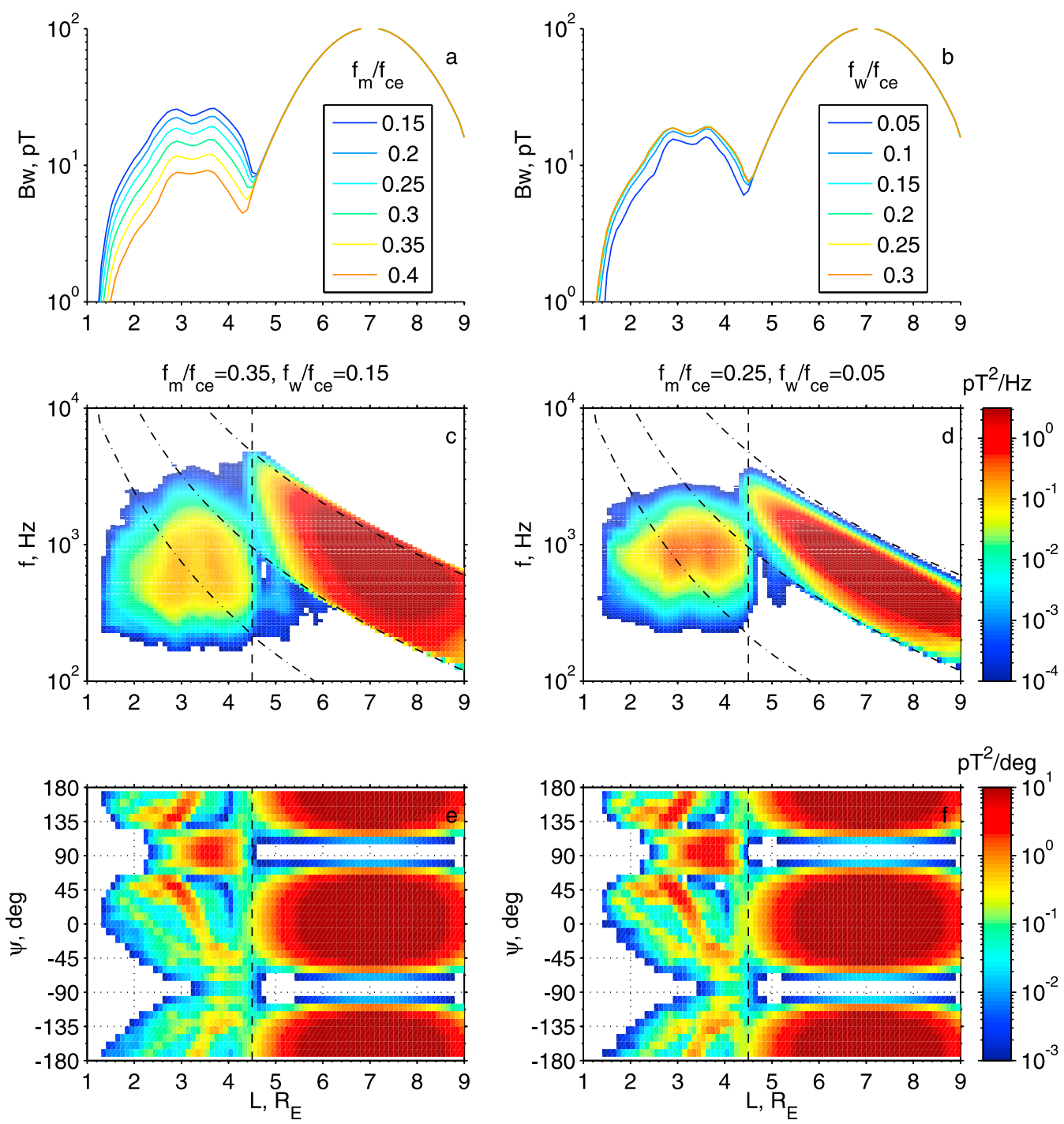

Figure 8. The dependence of equatorial hiss emission on the frequency distribution of chorus emission. (a) The equatorial hiss amplitude profile for varying chorus peak wave frequency $f_{m} / f_{\text {ce }}$ (color-coded). The (c) frequency and (e) wave normal angle spectrum of equatorial wave emission for $f_{m} / f_{\text {ce }}=0.35$. (b, d, and f) The same format as Figures $8 \mathrm{a}, 8 \mathrm{c}$, and $8 \mathrm{e}$ except for varying frequency width $f_{w} / f_{\text {ce. }}$. Figures $8 \mathrm{~d}$ and $8 \mathrm{f}$ show the frequency and wave normal angle spectrum of equatorial wave emission for $f_{w} / f_{\text {ce }}=0.05$. The spectrum for the nominal case is shown in Figures $5 \mathrm{~d}$ and $5 \mathrm{f}$ as comparison.

cases, the simulated hiss exhibits the two peaks, with the inner peak at intermediate wave normal angles near $45^{\circ}$ and $135^{\circ}$ and the outer peak at very oblique wave normal angles near $90^{\circ}$.

\subsection{Variation in Chorus Frequency Distribution}

[22] Figure 8 shows the response of hiss to the variation of the frequency distribution of the chorus waves. The effects of varying $f_{m}$ and $f_{w}$ are shown in Figures $8 \mathrm{a}$ and $8 \mathrm{~b}$, respectively. The wave frequency and wave normal distribution for the case $f_{m} / f_{\text {ce }}=0.35$ and $f_{w} / f_{\text {ce }}=0.15$ are shown in Figures $8 \mathrm{c}$ and $8 \mathrm{e}$ respectively, and those distributions for the case $f_{m} / f_{\text {ce }}=0.25$ and $f_{w} / f_{\text {ce }}=0.05$ are shown in Figures $8 \mathrm{~d}$ and $8 \mathrm{f}$. As the peak frequency $f_{m}$ increases, the hiss intensity decreases due to the weakening power at the lower frequency range of chorus, which is shown to contribute to most of hiss wave intensity (Figure 4c). Although the change of $f_{w}$ does not change the hiss amplitude significantly, it introduces a change in frequency spectrum. The narrower frequency distribution of chorus results in weaker wave power at the lower frequency portion of chorus emission so that hiss emission below $f<700 \mathrm{~Hz}$ is suppressed (Figure 8d). Therefore, the wave frequency distribution of hiss intensity becomes concentrated at $700 \mathrm{~Hz}-1000 \mathrm{~Hz}$. However, the wave normal angle distribution does not show a significant change except for the absolute intensity (comparing Figures $8 \mathrm{e}$ and $8 \mathrm{f}$ with Figure $5 \mathrm{f}$ ).

[23] Hiss also responds to the change in the lower $\left(f_{1}\right)$ and upper $\left(f_{2}\right)$ limit frequency of source chorus. Figures $9 \mathrm{a}$ and $9 \mathrm{~b}$ show the effects of varying $f_{1}$ and $f_{2}$, respectively. The resultant equatorial wave frequency spectrum and wave normal angle spectrum for the case $f_{1} / f_{\text {ce }}=0.15$ and $f_{2} / f_{\text {ce }}=0.5$ are shown in Figures $9 \mathrm{c}$ and 9e respectively, while the spectra for the case $f_{1} / f_{\text {ce }}=0.1$ and $f_{2} / f_{\text {ce }}=0.4$ are 

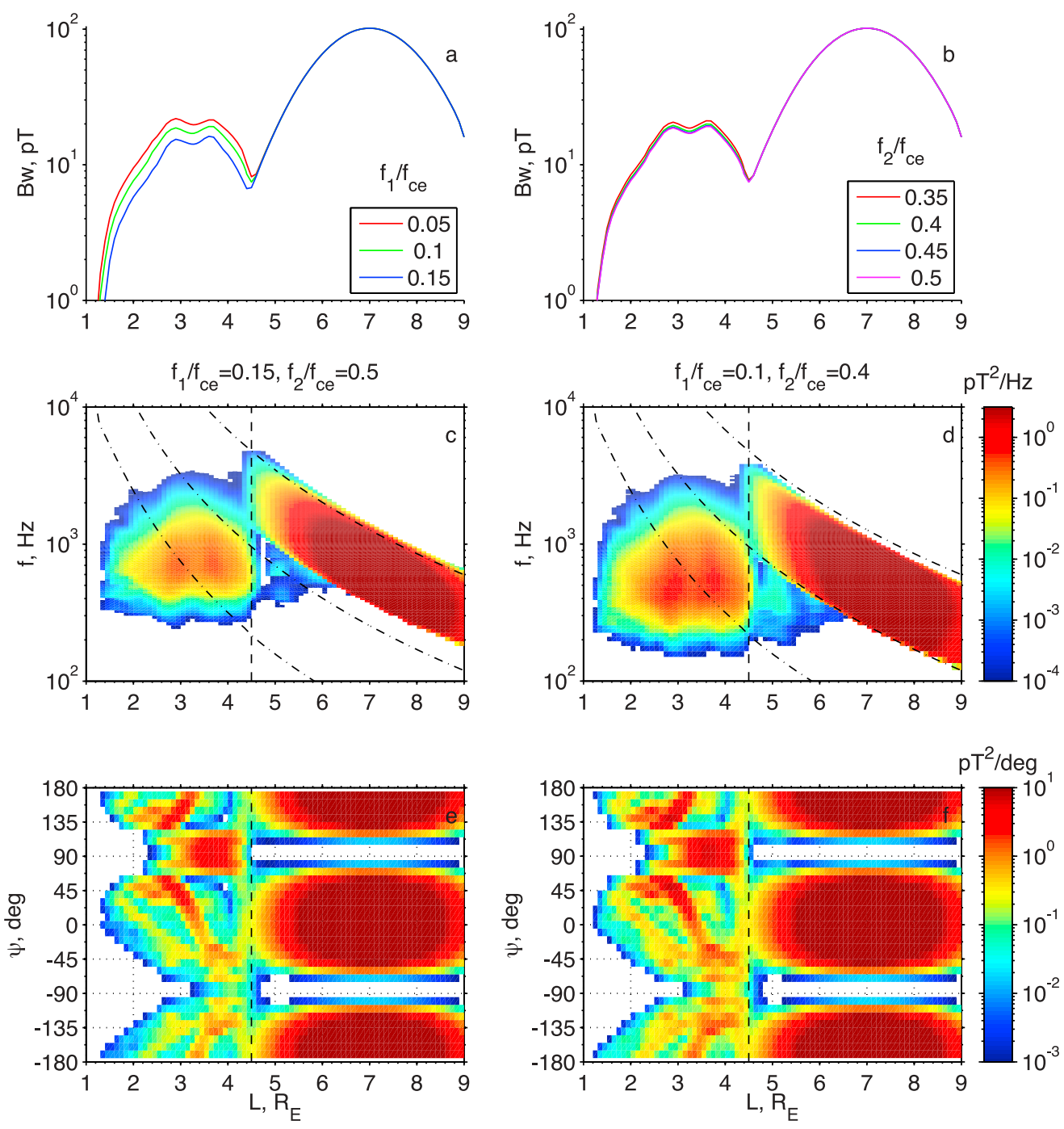

Figure 9. The dependence of equatorial hiss emission on the cutoff frequencies of chorus emission. (a) The equatorial hiss amplitude profile for varying lower cutoff frequency $f_{1} / f_{\text {ce }}$ (color-coded). The (c) frequency and (e) wave normal angle spectrum of equatorial wave emission for $f_{1} / f_{\mathrm{ce}}=0.15$. (b, $\mathrm{d}$, and f) The same format as Figures $9 \mathrm{a}, 9 \mathrm{c}$, and $9 \mathrm{e}$ except for varying upper cutoff frequencies $f_{2} / f_{\text {ce. }}$. Figures $9 \mathrm{~d}$ and $9 \mathrm{f}$ show the frequency and wave normal angle spectrum of equatorial wave emission for $f_{2} / f_{\mathrm{ce}}=0.4$. The spectrum for the nominal case is shown in Figures $5 \mathrm{~d}$ and $5 \mathrm{f}$ as comparison.

shown in Figures 9d and 9f respectively. As the lower limit frequency $f_{1}$ increases, the hiss intensity decreases because the chorus power at lower frequency is the primary source of the hiss emission (Figure 4c). It is evident that hiss intensity below $500 \mathrm{~Hz}$ is reduced for the case $f_{1}=0.15 f_{\text {ce }}$ (Figure 9c), compared with the case $f_{1}=0.1 f_{\text {ce }}$ (Figure 5d). The lower cutoff frequency of the chorus emissions is also responsible for the formation of a sharp lower cutoff frequency of the hiss emission, which has been reported in earlier observations [e.g., Smith et al., 1974]. The change in the upper cutoff frequency, which affects mostly chorus wave intensities near this upper limit frequency, does not affect the hiss intensity significantly. Both the frequency spectrum and wave normal distribution for the case $f_{2}=0.4 f_{\text {ce }}$ (Figures 9d and 9f) resemble those of $f_{2}=0.5 f_{\text {ce }}$ (Figures $5 \mathrm{~d}$ and 5f), respectively. As shown in Figure 4c, the chorus emission at frequency $>0.3 f_{\text {ce }}$ contributes little $(<3 \%)$ to the hiss intensity.

[24] Based on the variation of the chorus distribution examined in our study (with fixed chorus peak amplitude of $100 \mathrm{pT}$ ), the maximum hiss amplitude varies from less than $1 \mathrm{pT}$ (for the $\psi_{w}=10^{\circ}$ case in Figure $7 \mathrm{~b}$ ) to $>30 \mathrm{pT}$ (for cases with $L_{\text {peak }}$ from 5 to 6 in Figure 5a). However, none of the simulations performed are able to reproduce the observed ratio of 0.88 between the peak statistical amplitude of hiss $(54 \mathrm{pT})$ to that of chorus $(61 \mathrm{pT})$, shown in Figure $1 \mathrm{~b}$. We attribute this discrepancy to the omission of wave amplification inside the plasmasphere, as discussed below.

\section{Conclusion and Discussion}

[25] We performed a detailed simulation of the origin of plasmaspheric hiss from chorus emissions on the dayside. 
The spectral properties of hiss are modeled by using the realistic properties of chorus as an input and evaluating the wave propagation characteristics and wave damping. The response of the hiss intensity to the change of chorus distribution in $L$-shell, wave normal angle $\psi$ and wave frequency is also investigated. Our principle conclusions are as follows.

[26] 1 . We identify the portion of chorus emission that dominantly contributes to hiss intensity as those waves excited within $\sim 3 R_{E}$ from the plasmapause, within a finite range of intermediate wave normal angles ranges $-30^{\circ}<\psi<$ $-60^{\circ}$, and at low frequencies $f \leq 0.3 f_{\text {ce. }}$.

[27] 2. We predict the formation of two peaks in the $L$-shell distribution of hiss, consistent with observations. The inner peak is associated with the preferential location where newly entering chorus rays cross the equatorial plane on their first passage through the plasmasphere. Subsequent evolution of the entering chorus emission inside the plasmasphere generates the outer peak. The outer peak is mainly attributed to the wave energy accumulation due to magnetospherically reflected waves.

[28] 3. Hiss intensity is strongly dependent on the distribution of the chorus source emission, especially the relative location with respect to the plasmapause, the wave normal distribution and the frequency distribution at lower frequency $<0.3 f_{\text {ce. }}$. As chorus wave power increases at the lower frequency range, the wave normal direction points progressively Earthward, and the peak chorus wave power shifts closer to the plasmapause, the resulting hiss intensity increases.

[29] 4. Despite considerable variability of the hiss intensity, the frequency distribution and especially the wave normal angle distribution is relatively robust. The simulated hiss distribution is confined to the frequency range $\sim 200 \mathrm{~Hz}-$ $2 \mathrm{kHz}$, which is consistent with typical observed hiss frequency range. Wave intensity of hiss mostly concentrates near the field-aligned direction in the inner plasmasphere, but becomes very oblique just inside the plasmapause.

[30] 5. The statistical analysis of THEMIS wave data reveals that the peak hiss amplitude is slightly weaker than (a factor of $12 \%$ less than) the chorus peak amplitude.

[31] 6. Our simulated hiss based on observed chorus is $\sim 15 \mathrm{~dB}$ lower than the observed hiss emission, suggesting that it is necessary to include the internal amplification (presumably due to cyclotron resonant interactions) to account for the observed hiss intensity.

[32] Although the $\sim 15 \mathrm{~dB}$ deficit in simulated hiss peak intensity can be partially accounted for by variability of chorus distribution, including spatial distribution, frequency distribution and wave normal angle distribution, even under optimum conditions a discrepancy of $\sim 10 \mathrm{~dB}$ remains, which must be resolved by other processes not included in the current modeling. We suggest that additional wave amplification interior to the plasmasphere is possibly important to address this discrepancy. Previous modeling [e.g., Church and Thorne, 1983] indicates the possibility of internal amplification comparable to $\sim 20 \mathrm{~dB}$. This was deemed too small to account for the observed hiss intensity alone, but is sufficient to account for the discrepancy if chorus acts as the dominant embryonic source for hiss. Injected plasma sheet electrons normally are confined outside their Alfven layer, which is outside of the plasmapause. However, radial diffusion, possibly associated with variation of convection electric field in the magnetosphere, can probably transport the energetic electrons with sufficient anisotropy into the outer plasmasphere [Li et al., 2010a], and thus provide the source of free energy for wave growth. Using the properties of energetic electrons observed on the THEMIS spacecraft, we plan to develop a model of such resonant electron flux inside the plasmasphere and evaluate such internal amplification in the future modeling.

[33] Variability of hiss intensity could arise simply due to the variability of magnetosphere system itself, e.g., the cold plasma distribution, which affects the wave propagation trajectories and thus controls the distribution of wave power carried by each individual chorus ray. The response of hiss to the cold plasma density distribution is investigated in paper II.

[34] Modeling of hiss spectrum relies on the ray tracing technique, which treats the propagation of wave energy of a single wave packet in a prescribed (i.e., prescribed plasma density and background magnetic field) medium. There are a few possible sources of uncertainty in the modeling. First, the model of smooth plasma density might not accurately represent in-situ plasma density observation, which usually shows small length-scale density fluctuations. Second source of inaccuracies comes from the decomposition of observed chorus wave emission into many plane wave packets, which involves how the wave energy distributes in wave frequency and wave normal angle. Third, the potential growth of hiss inside the plasmasphere is not treated as mentioned above. Fourth, uncertainty might also come from the statistics of chorus emission and Landau damping electron fluxes derived from about 3 years THEMIS observation. Although our current model can not simulate fine structure of hiss emission, it is capable of reproducing many bulk features of hiss emission as discussed above.

[35] Two-dimensional ray tracing is a reasonable assumption in the prenoon sector where the azimuthal density gradient is not significant. Chorus emission in this sector is a primary source of the hiss emission in the same local times. However, Chen et al. [2009] have demonstrated that chorus propagation in the afternoon sectors, involving the large azimuthal density gradient associated with storm-time plumes, requires the consideration of three dimensional ray tracing. The $3 \mathrm{D}$ ray tracing links the chorus emission in the prenoon sector to the strong hiss inside the plume, and also potentially to weak hiss emission on the nightside, where chorus emission can not access to the plasmasphere directly in local nightside meridian plane. We plan to extend our 2D model to $3 \mathrm{D}$ in the future to understand the global source of hiss.

[36] Acknowledgments. This research was supported by NSF grant AGS-0840178 and NASA grant NNX08A135G. We acknowledge NASA contract NAS5-020P9 and V. Angelopoulos for use of data from the THEMIS mission, A. Roux and O. Le Contel for use of SCM data. The authors also thank World Data Center for Geomagnetism, Kyoto for providing the $\mathrm{AE}$ index.

[37] Robert Lysak thanks the reviewers for their assistance in evaluating this paper.

\section{References}

Abel, B., and R. M. Thorne (1998a), Electron scattering loss in Earth's inner magnetosphere: 1. Dominant physical processes, J. Geophys. Res. 103, 2385-2396, doi:10.1029/97JA02919.

Abel, B., and R. M. Thorne (1998b), Electron scattering loss in Earth's inner magnetosphere: 2. Sensitivity to model parameters, J. Geophys. Res., 103, 2397-2408, doi:10.1029/97JA02920. 
Albert, J. M. (1994), Quasi-linear pitch angle diffusion coefficients: Retaining high harmonics, J. Geophys. Res., 99(A12), 23,741-23,745, doi:10.1029/94JA02345.

Angelopoulos, V. (2008), The THEMIS mission, Space Sci. Rev., 141, 5-34, doi:10.1007/s11214-008-9336-1.

Bortnik, J., R. M. Thorne, and N. P. Meredith (2007), Modeling the propagation characteristics of chorus using CRRES suprathermal electron fluxes, J. Geophys. Res., 112, A08204, doi:10.1029/2006JA012237.

Bortnik, J., R. M. Thorne, and N. P. Meredith (2008), The unexpected origin of plasmaspheric hiss from discrete chorus emissions, Nature, 452 62-66, doi:10.1038/nature06741.

Bortnik, J., W. Li, R. M. Thorne, V. Angelopoulos, C. Cully, J. Bonnell, O. Le Contel, and A. Roux (2009), An observation linking the origin of plasmaspheric hiss to discrete chorus emissions, Science, 324, 775-778, doi:10.1126/science.1171273.

Bortnik, J., L. Chen, W. Li, R. M. Thorne, and R. B. Horne (2011a), Modeling the evolution of chorus waves into plasmaspheric hiss, J. Geophys. Res., 116, A08221, doi:10.1029/2011JA016499.

Bortnik, J., L. Chen, W. Li, R. M. Thorne, N. P. Meredith, and R. B. Horne (2011b), Modeling the wave power distribution and characteristics of plasmaspheric hiss, J. Geophys. Res., 116, A12209, doi:10.1029/ 2011JA016862.

Breneman, A. W., C. A. Kletzing, J. Pickett, J. Chum, and O. Santolik (2009), Statistics of multispacecraft observations of chorus dispersion and source location, J. Geophys. Res., 114, A06202, doi:10.1029/ 2008JA013549.

Burton, R. K., and R. E. Holzer (1974), The origin and propagation of chorus in the outer magnetosphere, J. Geophys. Res., 79, 1014-1023, doi:10.1029/JA079i007p01014.

Carpenter, D. L., and R. R. Anderson (1992), An ISEE/Whistler mode of equatorial electron density in the magnetosphere, J. Geophys. Res., 97(A2), 1097-1108.

Chappell, C. R., K. K. Harris, and G. W. Sharp (1970), A study of the influence of magnetic activity on the location of the plasmapause as measured by OGO 5, J. Geophys. Res., 75, 50-56, doi:10.1029/JA075i001p00050.

Chen, L., J. Bortnik, R. M. Thorne, R. B. Horne, and V. K. Jordanova (2009), Three-dimensional ray tracing of VLF waves in a magnetospheric environment containing a plasmaspheric plume, Geophys. Res. Lett., 36 , L22101, doi:10.1029/2009GL040451.

Chen, L., R. M. Thorne, V. K. Jordanova, C.-P. Wang, M. Gkioulidou, L. Lyons, and R. B. Horne (2010), Global simulation of EMIC wave excitation during the 21 April 2001 storm from coupled RCM-RAMHOTRAY modeling, J. Geophys. Res., 115, A07209, doi:10.1029/ 2009JA015075

Chen, L., J. Bortnik, R. M. Thorne, W. Li, and R. B. Horne (2012), Modeling the properties of plasmaspheric hiss: 2. Dependence on the plasma density distribution, J. Geophys. Res., 117, A05202, doi:10.1029/ 2011JA017202.

Church, S. R., and R. M. Thorne (1983), On the origin of plasmaspheric hiss: Ray path integrated amplification, J. Geophys. Res., 88, 7941-7957, doi:10.1029/JA088iA10p07941.

Cully, C. M., R. E. Ergun, K. Stevens, A. Nammari, and J. Westfall (2008), The THEMIS Digital Fields Board, Space Sci. Rev., 141, 343-355, doi: $10.1007 / \mathrm{s} 11214-008-9417-1$.

Denton, R. E., J. Goldstein, and J. D. Menietti (2002), Field line dependence of magnetospheric electron density, Geophys. Res. Lett., 29(24), 2205, doi:10.1029/2002GL015963.

Denton, R. E., K. Takahashi, I. A. Galkin, P. A. Nsumei, X. Huang, B. W. Reinisch, R. R. Anderson, M. K. Sleeper, and W. J. Hughes (2006), Distribution of density along magnetospheric field lines, J. Geophys. Res. 111, A04213, doi:10.1029/2005JA011414

Draganov, A. B., U. S. Inan, V. S. Sonwalkar, and T. F. Bell (1992), Magnetospherically reflected whistlers as a source of plasmaspheric hiss, Geophys. Res. Lett., 19, 233-236, doi:10.1029/91GL03167.

Fu, H. S., J. Tu, J. B. Cao, P. Song, B. W. Reinisch, D. L. Gallagher, and B. Yang (2010), IMAGE and DMSP observations of a density trough inside the plasmasphere, J. Geophys. Res., 115, A07227, doi:10.1029/2009JA015104

Goldstein, B. E., and B. T. Tsurutani (1984), Wave normal directions of chorus near the equatorial source region, J. Geophys. Res., 89, 2789-2810, doi:10.1029/JA089iA05p02789.

Goldstein, J., M. Spasojevic, P. H. Reiff, B. R. Sandel, W. T. Forrester, D. L. Gallagher, and B. W. Reinisch (2003), Identifying the plasmapause in IMAGE EUV data using IMAGE RPI in situ steep density gradients, J. Geophys. Res., 108(A4), 1147, doi:10.1029/2002JA009475.

Grebowsky, J. M. (1970), Model study of plasmapause motion, J. Geophys. Res., 75, 4329-4333, doi:10.1029/JA075i022p04329.
Green, J. L., S. Boardsen, L. Garcia, W. W. L. Taylor, S. F. Fung, and B. W. Reinisch (2005), On the origin of whistler mode radiation in the plasmasphere, J. Geophys. Res., 110, A03201, doi:10.1029/2004JA010495.

Haque, N., M. Spasojevic, O. Santolík, and U. S. Inan (2010), Wave normal angles of magnetospheric chorus emissions observed on the Polar spacecraft, J. Geophys. Res., 115, A00F07, doi:10.1029/2009JA014717.

Harris, K. K. (1974), The measurement of cold ion densities in the plasma trough, J. Geophys. Res., 79, 4654-4660, doi:10.1029/JA079i031p04654.

Hayakawa, M., and S. S. Sazhin (1992), Mid-latitude and plasmaspheric HISS-A review, Planet. Space Sci., 40, 1325-1338, doi:10.1016 0032-0633(92)90089-7.

Horne, R. B. (1989), Path-integrated growth of electrostatic waves: The generation of terrestrial myriametric radiation, J. Geophys. Res., 94(A7), 8895-8909.

Huang, C. Y., and C. K. Goertz (1983), Ray-tracing studies and pathintegrated gains of ELF unducted whistler mode waves in the Earth's magnetosphere, J. Geophys. Res., 88, 6181-6187, doi:10.1029/ JA088iA08p06181.

Huang, C. Y., C. K. Goertz, and R. R. Anderson (1983), A theoretial study of plasmaspheric hiss generation, J. Geophys. Res., 88, 7927-7940, doi:10.1029/JA088iA10p07927.

Jordanova, V. K., R. M. Thorne, W. Li, and Y. Miashi (2010), Excitation of whistler-mode chorus from global ring current simulations, J. Geophys. Res., 115, A00F10, doi:10.1029/2009JA014810.

Kitamura, N., A. Shinbori, Y. Nishimura, T. Ono, M. Iizima, and A. Kumamoto (2009), Seasonal variations of the electron density distribution in the polar region during geomagnetically quiet periods near solar maximum, J. Geophys. Res., 114, A01206, doi:10.1029/2008JA013288.

Lauben, D. S., U. S. Inan, T. F. Bell, and D. A. Gurnett (2002), Source characteristics of ELF/VLF chorus, J. Geophys. Res., 107(A12), 1429, doi:10.1029/2000JA003019.

Le Contel, O., et al. (2008), First results of the themis search coil magnetometers, Space Sci. Rev., 141, 509-534, doi:10.1007/s11214-008-9371-y. Li, W., R. M. Thorne, V. Angelopoulos, J. Bortnik, C. M. Cully, B. Ni, O. LeContel, A. Roux, U. Auster, and W. Magnes (2009), Global distribution of whistler-mode chorus waves observed on the THEMIS spacecraft, Geophys. Res. Lett., 36, L09104, doi:10.1029/2009GL037595.

Li, W., R. M. Thorne, J. Bortnik, Y. Nishimura, V. Angelopoulos, L. Chen, J. P. McFadden, and J. W. Bonnell (2010a), Global distributions of suprathermal electrons observed on THEMIS and potential mechanisms for access into the plasmasphere, J. Geophys. Res., 115, A00J10, doi: $10.1029 / 2010 \mathrm{JA} 015687$.

Li, W., et al. (2010b), THEMIS analysis of observed equatorial electron distributions responsible for the chorus excitation, J. Geophys. Res., 115, A00F11, doi:10.1029/2009JA014845.

Li, W., R. M. Thorne, J. Bortnik, Y. Y. Shprits, Y. Nishimura V. Angelopoulos, C. Chaston, O. L. Contel, and J. W. Bonnell (2011a), Typical properties of rising and falling tone chorus waves, Geophys. Res. Lett., 38, L14103, doi:10.1029/2011GL047925.

Li, W., J. Bortnik, R. M. Thorne, and V. Angelopoulos (2011b), Global distribution of wave amplitudes and wave normal angles of chorus waves using THEMIS wave observations, J. Geophys. Res., 116, A12205, doi:10.1029/2011JA017035.

Lyons, L. R., and R. M. Thorne (1973), Equilibrium structure of radiation belt electrons, J. Geophys. Res., 78, 2142-2149, doi:10.1029/ JA078i013p02142.

McFadden, J. P., C. W. Carlson, D. Larson, M. Ludlam, R. Abiad, B. Elliott, P. Turin, M. Marckwordt, and V. Angelopoulos (2008), The THEMIS ESA plasma instrument and in-flight calibration, Space Sci. Rev., 141, 277-302, doi:10.1007/s11214-008-9440-2

Meredith, N. P., R. M. Thorne, R. B. Horne, D. Summers, B. J. Fraser and R. R. Anderson (2003), Statistical analysis of relativistic electron energies for cyclotron resonance with EMIC waves observed on CRRES J. Geophys. Res., 108(A6), 1250, doi:10.1029/2002JA009700.

Meredith, N. P., R. B. Horne, R. M. Thorne, D. Summers, and R. R. Anderson (2004), Substorm dependence of plasmaspheric hiss, J. Geophys. Res., 109, A06209, doi:10.1029/2004JA010387.

Meredith, N. P., R. B. Horne, S. A. Glauert, R. M. Thorne, D. Summers, J. M. Albert, and R. R. Anderson (2006), Energetic outer zone electron loss timescales during low geomagnetic activity, J. Geophys. Res., 111, A05212, doi:10.1029/2005JA011516.

Meredith, N. P., R. B. Horne, S. A. Glauert, and R. R. Anderson (2007), Slot region electron loss timescales due to plasmaspheric hiss and lightninggenerated whistlers, J. Geophys. Res., 112, A08214, doi:10.1029/ 2007JA012413.

Meredith, N. P., R. B. Horne, S. A. Glauert, D. N. Baker, S. G. Kanekal, and J. M. Albert (2009), Relativistic electron loss timescales in the slot region, J. Geophys. Res., 114, A03222, doi:10.1029/2008JA013889. 
Reinisch, B. W., P. Nsumei, X. Huang, and D. K. Bilitza (2007), Modeling the F2 topside and plasmasphere for IRI using IMAGE/RPI and ISIS data, Adv. Space Res., 39, 731-738, doi:10.1016/j.asr.2006.05.032.

Richards, P. G., and D. G. Torr (1985), Seasonal, diurnal, and solar cyclical variations of the limiting $\mathrm{H}^{+}$flux in the Earth's topside ionosphere, J. Geophys. Res., 90, 5261-5268, doi:10.1029/JA090iA06p05261.

Roux, A., O. Le Contel, C. Coillot, A. Bouabdellah, B. de la Porte, D. Alison, S. Ruocco, and M. Vassal (2008), The search coil magnetometer for THEMIS, Space Sci. Rev., 141, 265-275, doi:10.1007/s11214008-9455-8.

Russell, C. T., R. E. Holzer, and E. J. Smith (1969), OGO 3 observations of ELF noise in the magnetosphere: 1. Spatial extent and frequency of occurrence, J. Geophys. Res., 74, 755-777, doi:10.1029/JA074i003p00755.

Santolík, O., D. A. Gurnett, J. S. Pickett, M. Parrot, and N. CornilleauWehrlin (2003), Spatio-temporal structure of storm-time chorus, J. Geophys. Res., 108(A7), 1278, doi:10.1029/2002JA009791.

Santolík, O., D. A. Gurnett, J. S. Pickett, J. Chum, and N. Cornilleau-Wehrlin (2009), Oblique propagation of whistler mode waves in the chorus source region, J. Geophys. Res., 114, A00F03, doi:10.1029/2009JA014586.

Smith, E. J., A. M. A. Frandsen, B. T. Tsurutani, R. M. Thorne, and K. W. Chan (1974), Plasmaspheric hiss intensity variations during magnetic storms, J. Geophys. Res., 79, 2507-2510, doi:10.1029/JA079i016p02507.

Sonwalkar, V. S., and U. S. Inan (1989), Lightning as an embryonic source of VLF hiss, J. Geophys. Res., 94, 6986-6994, doi:10.1029/ JA094iA06p06986.
Summers, D., B. Ni, N. P. Meredith, R. B. Horne, R. M. Thorne, M. B. Moldwin, and R. R. Anderson (2008), Electron scattering by whistlermode ELF hiss in plasmaspheric plumes, J. Geophys. Res., 113, A04219, doi:10.1029/2007JA012678

Thorne, R. M., E. J. Smith, R. K. Burton, and R. E. Holzer (1973), Plasmaspheric hiss, J. Geophys. Res., 78, 1581-1596, doi:10.1029/ JA078i010p01581.

Tsurutani, B. T., E. J. Smith, and R. M. Thorne (1975), Electromagnetic hiss and relativistic electron losses in the inner zone, J. Geophys. Res., 80, 600-607, doi:10.1029/JA080i004p00600.

Tsurutani, B. T., O. P. Verkhoglyadova, G. S. Lakhina, and S. Yagitani (2009), Properties of dayside outer zone chorus during HILDCAA events: Loss of energetic electrons, J. Geophys. Res., 114, A03207, doi:10.1029/2008JA013353.

Wang, C., Q. Zong, F. Xiao, Z. Su, Y. Wang, and C. Yue (2011), The relations between magnetospheric chorus and hiss inside and outside the plasmasphere boundary layer: Cluster observation, J. Geophys. Res., 116 , A07221, doi:10.1029/2010JA016240.

J. Bortnik, L. Chen, W. Li, and R. M. Thorne, Department of Atmospheric Sciences, University of California, Los Angeles, CA 90024, USA. (clj@atmos.ucla.edu)

R. B. Horne, British Antarctic Survey, Natural Environment Research Council, Cambridge CB3 0ET, UK. 